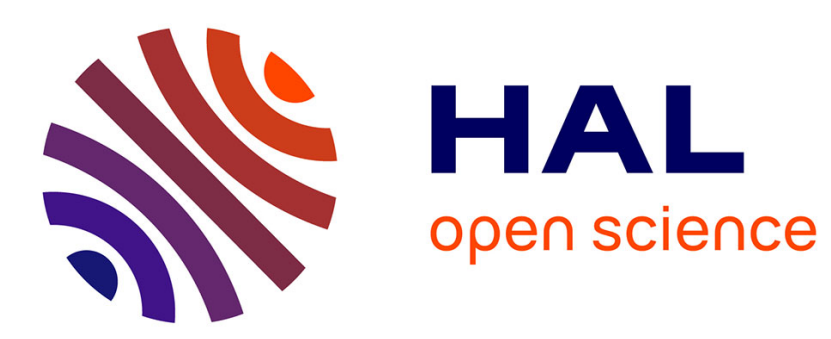

\title{
Enhancing the enantioselectivity of CALB by substrate imprinting: A combined experimental and molecular dynamics simulation model study
}

Ludovic Chaput, Zsuzsanna Marton, Philippe Pineau, Lisiane Domon, Vinh Tran, Marianne Graber

\section{To cite this version:}

Ludovic Chaput, Zsuzsanna Marton, Philippe Pineau, Lisiane Domon, Vinh Tran, et al.. Enhancing the enantioselectivity of CALB by substrate imprinting: A combined experimental and molecular dynamics simulation model study. Journal of Molecular Catalysis B: Enzymatic, 2012, 84, pp.55-61. hal-00789671

\section{HAL Id: hal-00789671 \\ https://hal.science/hal-00789671}

Submitted on 18 Feb 2013

HAL is a multi-disciplinary open access archive for the deposit and dissemination of scientific research documents, whether they are published or not. The documents may come from teaching and research institutions in France or abroad, or from public or private research centers.
L'archive ouverte pluridisciplinaire HAL, est destinée au dépôt et à la diffusion de documents scientifiques de niveau recherche, publiés ou non, émanant des établissements d'enseignement et de recherche français ou étrangers, des laboratoires publics ou privés. 


\title{
Enhancing the Enantioselectivity of CALB by substrate imprinting: an experimental and molecular dynamics simulation study.
}

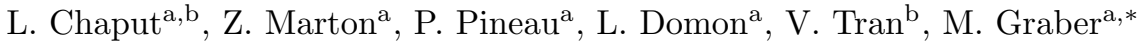 \\ ${ }^{a}$ Université La Rochelle, UMR 6250 LIENSs CNRS - ULR, Pôle Sciences et Technologies, Bâtiment Marie Curie, Avenue \\ Michel Crépeau, 17042 La Rochelle, France \\ ${ }^{b}$ Unité Biotechnologie, Biocatalyse et Biorégulation (U3B), UMR CNRS 6204 - Faculté des Sciences et des Techniques de \\ Nantes, 2, rue de la Houssinière, BP 92208 44322 Nantes Cedex 3, France
}

\begin{abstract}
Kinetic resolution of pentan-2-ol by CALB catalyzed enantioselective transesterification, with various alkylpropanoate acyl donors, was studied in a solid-gas reactor. Results show that the leaving alkoxy group influences the enantiomeric ratio of the reaction. Resolution of pentan-2-ol with methyl propanoate gives an enantiomeric ratio of 62 . Esters with longer linear alkyl chains, from ethyl to pentyl propanoate give higher enantiomeric ratios, comprised between 103 and 117. Enantiopure ester (R)-1-methylpentyl propanoate increases the enantiomeric ratio to 140 compared with $E=120$ for the racemic mixture. In contrast, enantiopure (S)-1-methylpentyl propanoate decreases the enantiomeric ratio to 72 . Our data support the notion of an imprinting effect or "ligand-induced enzyme memory" caused by the shape of the leaving alcohol.

To simulate the imprinting effect caused by the alkoxy part of the acyl donor, molecular modeling studies were performed with both $(R)$ - and $(S)$ - enantiopure 1-methylpentyl propanoate. To investigate how the first step of the reaction, through the first tetrahedral intermediate, affects the enzyme conformation depending on the enantiopure ester substrate used, 20 ns molecular dynamics simulations were carried out. Clustering analysis was done to study relevant conformations of the systems. Differences in the global conformation of the enzyme between systems with $R$ or $S$ enantiomers were not observed. Interestingly however, orientation of the partially buried side chain for Ile285 was affected. This could explain the increased enantiomeric ratio observed with the substrate ester $(R)$-1-methylpentyl propanoate due to an improved $(R)$-pentan-2ol/enzyme interaction.
\end{abstract}

Keywords: enantioselectivity, imprinting effect, kinetics, Candida antarctica lipase B, molecular modeling

\footnotetext{
${ }^{*}$ Corresponding author. Tel: +33 5464586 30; fax: +33 546458265 .

Email address: mgraber@univ-lr.fr (M. Graber)
} 


\section{Introduction}

Lipases are $\alpha / \beta$ hydrolases (EC 3.1.1.3) which catalyze the hydrolysis of triglycerides in vivo. They can also form ester bonds under reverse hydrolytic conditions, which enables them to catalyze esterification and transesterification reactions. Furthermore, they are enantioselective catalysts useful in the synthesis of pharmaceutical intermediates and fine chemicals. Lipase B from Candida antarctica, CALB, has found widespread applications in the enantioselective synthesis of bioactive molecules and in the resolution of racemic mixtures, due to its high stability in organic media and its large-scale availability [1].

CALB catalyzed resolution of secondary alcohols by transesterification with esters as acyl donors, occurs through a Ping Pong Bi Bi mechanism, which includes two steps. The first is the acylation of the enzyme by the ester substrate, to yield the acyl-enzyme intermediate and the release of the first product, an alcohol formed with the alkoxy group. In the second step, the chiral alcohol interacts with the acyl-enzyme to form a new binary complex and the second product ester.

In the active site of CALB, the acyl and alcohol substrate moieties bind in a hairpin orientation. It is thus not surprising that the acyl chain of the ester interacts with the alcohol and consequently influences the chiral discrimination of alcohols. However it is unexpected that the leaving group, which is the alkoxy part of the ester, also influences the enantiomeric ratio.

The hypothesis of "molecular imprinting effect" has been proposed by several authors to explain such modification of enzyme selectivity or activity $[2,3,4]$. The enzyme molds its active site structure around the imprint molecule and remains "trapped" in this conformation until the substrate enters.

In 2000, Lee et al. [5] described a new approach to enhance lipase enantioselectivity by the "substrate matching" strategy. Resolution of three different secondary alcohols with three different acyl donors by lipasecatalyzed transesterification with Candida antarctica and Pseudomonas cepacia lipases were performed. Results demonstrated that the enantioselectivity of lipases was maximized by using acyl donor and alcohol substrates which matched well. The hypothesis of the "enzyme memory" induced by the acyl donors active site moulding in the first step of the reaction was proposed.

In the present work, we experimentally highlight the significant influence of the alkoxy part of the ester acyl donor on the enantiomeric ratio, for the resolution of pentan-2-ol by CALB. We then established the full kinetic model for a Ping-Pong Bi Bi mechanism with two competing chiral alcohol substrates, in order to verify that the differences in enantiomeric ratio, obtained with different acyl donors, did not simply arise from differences in reaction rates occurring during the acylation step, with the different esters. Our data from both experimental and kinetic studies support the hypothesis of molecular imprinting. We then looked for structural changes using molecular modeling methods.

Molecular modeling is a useful tool to provide a rational explanation of experimental data. In 2010, Lousa et al. provided a structural explanation for the imprinting effect [6] observed with pre-treated subtisilin 
by co-lyophilisation with an inhibitor in the active site, using a molecular modeling approach. Results showed that in the presence of the inhibitor, the active site was maintained in an open conformation which was stable in hexane solvent, in contrast to simulation with "untreated" enzyme. Here, 20 ns molecular dynamics simulations were carried out to study how the first step of the reaction, through the first tetrahedral intermediate, affects the enzyme conformation, depending on the enantiopure ester substrate used.

\section{Experimental Section}

\subsection{Chemicals}

Substrates and other chemicals were purchased from SigmaAldrich-Fluka Chemical Co. They were of the highest purity available (98\% minimum) and checked by gas chromatography before use. Substrates were dried by distillation under argon prior to use and stored under argon atmosphere and over molecular sieves. Solvents were purchased from Carlo Erba. Racemic 1-methylpentylpropanoate was synthesized from the corresponding alcohol and propanoic anhydride in pyridine at room temperature [7].

(R)-1-methylpentyl propanoate was obtained by enzymatic resolution from vinyl propionate and hexan2-ol using CALB Novozym ${ }^{\circledR} 435$ in heptane solvent at $35^{\circ} \mathrm{C}$, ee $e_{p}$ enantiomeric excess of ester product was $99.3 \%$. Enriched hexan-2-ol in $S$ form taken from the previous reaction was used after purification by chromatography on silica gel (eluent EP/AcOEt: 95/5), then esterification with anhydrid propionic was done to obtain 1-(S)-methylpentyl propanoate.

\subsection{Enzyme used for kinetic studies}

CALB was produced in the methylotropic yeast Pichia pastoris and was expressed extracellularly and purified from the medium by hydrophobic interaction chromatography, followed by gel filtration $[8,9]$. Enzyme adsorption was performed onto 60/80 mesh Chromosorb P AW DMCS (acid washed dimethylchlorosilanized) (Varian, France). In a typical adsorption procedure for solid/gas catalysis, enzyme (0.106 mg) was dissolved in sodium phosphate buffer ( $\mathrm{pH} 7.5,10 \mathrm{mM})$, and dry Chromosorb P AW DMCS (1 g) was added to the solution. The amount of immobilized enzyme was determined by measuring absorbance at $280 \mathrm{~nm}$, by taking a molar extinction coefficient equal to $40690 \mathrm{M}^{-1} \cdot \mathrm{cm}^{-1}$. After vigorous shaking, the preparation was left for 1 week under vacuum and over $\mathrm{P}_{2} \mathrm{O}_{5}$ at room temperature.

\subsection{Enzymatic reactions}

Initial rate of reaction measurements were performed at $70{ }^{\circ} \mathrm{C}$ in a solid-gas reactor as previously described [10]. Thermodynamic activities for ester and alcohol substrates were respectively $a_{e s t e r}=0.1$ and $a_{\text {alcohol }}=0.05$. Reactions were carried out in anhydrous conditions. The amount of enzyme comprised between 20 and $200 \mathrm{mg}$, depending on the acyl donor used. The total flow was equal to $900 \mu \mathrm{mol}^{\mathrm{mol}}{ }^{-1}$. 


\section{4. $G C$ analysis}

Quantitative analysis of reaction products were conducted using a $7890 \mathrm{GC}$ system from Agilent for the analysis of ester products (R)-1- and (S)-1-methylbutyl propanoate $\left(55{ }^{\circ} \mathrm{C} 15 \mathrm{~min}, 3{ }^{\circ} \mathrm{C} \cdot \mathrm{min}^{-1}, 85^{\circ} \mathrm{C} 5\right.$ min), at a flow rate of $1.5 \mathrm{ml}$.min-1 with a Chirasil-Dex CB (25 m, $0.25 \mathrm{~mm}$ i.d., $0.25 \mu \mathrm{m} \beta$-cyclodextrin, Chrompack, France) column. Products were detected by FID and quantified using HP Chemstation software.

\subsection{Enantioselectivity measurements}

Enantiomeric ratio values for the different kinetics reported in the Results section, were obtained in our laboratory, by measuring the ratio of initial reaction rates for ester products synthesis [11], in a continuous solid-gas reactor with different acyl donors, and immobilized CALB, as previously described $[9,12]$.

\section{Computational Methods}

\subsection{Setup of the system}

The starting CALB enzyme was the $\mathrm{R}=1.55 \AA$ crystallographic structure solved by Uppenberg et al. [13] (PDB entry 1TCA). To evaluate the effect of the ester substrate on the enzyme structure during the first step of the reaction path, the two tetrahedral intermediates, obtained in the reaction with $R$ or $S$ 1-methylpentyl propanoate were modelized. The choice of studying the intermediates, instead of free substrates, in the active site was done in order to prevent the substrates from getting out of active site, observed several times in the case of subtilisin by Lousa et al. [6]. Furthermore, the formation of the tetrahedral intermediate may have more impact on the structure conformation, because its formation requires the crossing of the energy barrier. Thus, three systems were modelized: free enzyme, enzyme with $R$ and $S$ tetrahedral intermediates.

A transition state analog crystal structure, obtained with phosphonate irreversible inhibitor (PDB entry 1LBS) was used to build the tetrahedral part of the reaction intermediate, to allow for the correct location of the central part of the tetrahedral intermediate. The acyl part is a propanoyl group. The negatively charged oxygen was oriented toward the oxyanion hole to establish hydrogen bonds with Thr40 and Gln106.

NAMD 2.7 program and the CHARMM22 all-atom force field were used. Calculations were done in an explicit water box (model TIP3P) with boundary conditions (15 $\AA$ between the enzyme and the edge of the box). A trajectory of 20 nanoseconds was done for each system. The thermodynamic ensemble is "isotherm-isobar" (NTP). The timestep was 2 fs and the SHAKE alghorithm was used to freeze bonds involving hydrogen atoms.

Force field parameters for the tetrahedral intermediate were taken from the literature [14]. These parameters were obtained from ab initio calculations and were specifically developed for CHARMM22 force field. Other parameters required for modeling the alkyl side chains of alcohols were defined by homology with available CHARMM22 parameters. 
First, water molecules surrounding the enzyme were minimized by 5000 iterations of the conjugate gradient, then the whole system was minimized with 10000 iterations using the same algorithm. The heat steps were carried out in $600 \mathrm{ps}$, starting from $50 \mathrm{~K}$ and going up to $300 \mathrm{~K}$, with a temperature incrementation of $1 \mathrm{~K}$ every $4 \mathrm{ps}$. An harmonic constraint of $5 \mathrm{kcal}^{\mathrm{mol}}{ }^{-1}$ was set up on the enzyme. The equilibration step is the succession of four short dynamics of 200 ps with a decreasing harmonic constraint $(5,3,0.5$ and 0.1 kcal.mol ${ }^{-1}$ ) followed by one nanosecond without constraint. Then, the production dynamic lasted for $20 \mathrm{~ns}$.

\subsection{Clustering analysis}

Clustering analysis provides a good overview of enzyme conformations. 2000 structures, extracted from the productive dynamics (one every $10 \mathrm{ps),} \mathrm{were} \mathrm{used} \mathrm{for} \mathrm{the} \mathrm{dynamic} \mathrm{analysis.} \mathrm{Using} \mathrm{the} \mathrm{VMD} \mathrm{program} \mathrm{[15],}$ RMSD (root-mean-square-deviation) matrices were calculated for the 2000 structures, one diagonal matrix with a size of 2000 by 2000 was obtained for each of the three systems.

RMSD was calculated on the backbone for residues 35 to 80, 100 to 240 and 260 to 290. Residues far from the active site were not included, because their mobility is not supposed to influence the active site conformation, which is the region putatively involved in the imprinting effect. In addition, terminal regions are highly mobile (and far from the active site for CALB) and over-influenced the RMSD matrix, therefore, they were not included. In the manner, we aimed to obtain conformational information specific to the rest of the structure and more particularly near the active site.

In a second step, RMSD matrix was calculated to focus on the residues of the active site. RMSD were based on every heavy atom, including side chains, of residues 103, 104, 106, 224, 187, 40, 42, 47, 278, 282, 285, and the backbone of residue 105 (due to the fact that the side chain changes for each system for this residue). This RMSD matrix was then used to process a hierarchical ascendant classification (HAC). The Ward method [16] was applied to the agglomerative steps used to build the dendrogram. Clustering analysis was carried out using the $\mathrm{R}$ statistical software [17]. The average structure of the two most representative structures from productive dynamics was chosen. Then, the closest structures to the average structure were extracted and used for analysis.

\section{Results and Discussion}

\subsection{Experimental results}

Enantiomeric ratios experimentally determined for the resolution of pentan-2-ol by transesterification with various alkoxy propanoates as acyl donors are presented in table 1. Resolution of pentan-2-ol with methyl propanoate displays an enantiomeric ratio of 62 . Esters with longer linear alkyl chains, from ethyl to pentyl propanoate give higher enantiomeric ratios up to 117. Enantiomeric ratios equal to 117, 103, 115 and 105 were found for ethyl propanoate, propyl propanoate, butyl propanoate and pentyl propanoate, 
respectively. Enantiomeric ratios for chiral esters with branched alkyl chains were also evaluated. The racemate 1-methylpropyl propanoate gives an $E$ value of 51 , which is quite close to the $E$ value (62) for methyl propanoate. Higher enantiomeric ratios were obtained with the longer racemate 1-ethylbutyl propanoate (ratio of 84) and with 1-methylpentyl propanoate, (ratio of 122).

Enantiomeric ratios with the enantiopure $(R)$-1-methylpentyl propanoate and (S)-1-methylpentyl propanoate, were measured and found to be 140 and 72, respectively. Thus, the enantiomeric form of the chiral ester substrate is essential for determining the enantioselectivity of the reaction. CALB displays enantiopreference for the $R$ alcohol [9, 18], and using an ester with the $R$ chiral form for the alkyl part increases the enantioselectivity compared to the racemate. In contrast, an ester with the $S$ chiral form for the alkyl part, results in decreased enantioselectivity. Therefore, the more the alkyl part of the chiral ester resembles the preferred enantiomer, $(R)$-pentan-2-ol, the higher the enantioselectivity attained. A similar observation was made by González-Sabín et al. [19], who obtained higher enantioselectivity when the alkoxy group of the acyl donor was structurally close to the amine to be resolved. As a consequence an improved resolution of $( \pm)$-cis-2-phenylcyclopentanamine was obtained with the leaving group ( \pm )-cisphenylcyclopentanol $(E$ value $=922)$, compared to $( \pm)$-trans-phenylcyclopentanol $(E$ value $=525)$.

Table 1: Enantiomeric ratio for CALB catalyzed transesterification involving pentan-2-ol with different alkyl propanoate esters, in solid-gaz reactor at $70^{\circ} \mathrm{C}$.

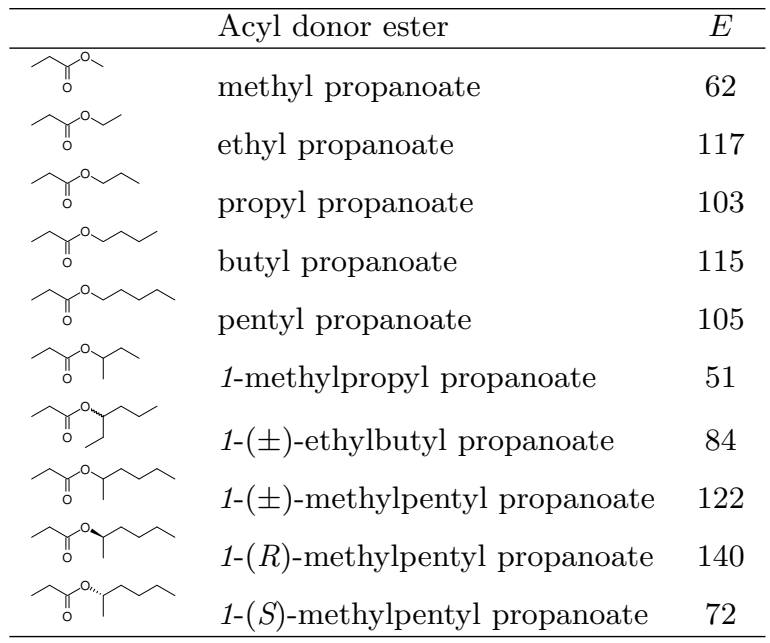

\subsection{Kinetic equation study}

To confirm whether the differences in enantioselectivity are due to an imprinting effect caused by the leaving alcohol, it was necessary to check that these differences, obtained using different acyl donors, do not simply arise from differences in reaction rates occurring during the acylation step with the different esters. The complete kinetic model for the Ping Pong Bi Bi mechanism, with two competing chiral alcohol substrates, was established. The enantiomeric ratio was then expressed as a function of individual catalytic 
rate constants of the reaction, in order to investigate whether the catalytic rate constants involved in the acylation step influence the $E$ value.

\subsubsection{Kinetic model determination}

The enantiomeric ratio is defined as the ratio of specificity constants for $R$ and $S$ enantiomers, according to the following formula: $E=\left(k_{\text {cat }}^{R} / K_{M}^{R}\right) /\left(k_{\text {cat }}^{S} / K_{M}^{S}\right)$. The kinetic parameter determination is straightforward in the case of a monosubstrate reaction following the classic Michaelis mechanism. The transesterification studied here corresponds to a much more complex kinetics system. It involves a first substrate ester and two competing second substrates, $R$ and $S$ forms of the secondary alcohol. It obeys a Ping Pong Bi Bi mechanism. Classic kinetic experiments provide apparent constants $K_{M}$ and $V_{\max }$, which are dependent on the catalytic rate constant of the first step of the mechanism. The Michaelis-Menton constant for the $R$ alcohol $K_{M}^{R}$ is thus equal to $k_{2}\left(k_{3}+k_{4}\right) / k_{3}\left(k_{4}+k_{2}\right)$ (figure 2), where $k_{2}$ depends on the leaving alcohol in the first reaction step. This observation could explain the enantiomeric ratio modification observed when the leaving alkoxy group changes. Therefore, the relationship between the acylation step rate and enantiomeric ratio is worth considering. Here, the full kinetic model for a Ping Pong Bi Bi mechanism involving one ester and two competiting alcohols substrates was defined. The kinetic equation was calculated using the King-Altman method and specificity constants were determined with the Cleland method.

We focused on the resolution of a racemic mixture of $R$ and $S$ enantiomeric forms of pentan-2-ol, through acyl transfer from an ester substrate. In the reaction model a second pathway for the reaction with the second enantiomer was added, as shown in figures 1 and 2, for Cleland and King-Altman representations.

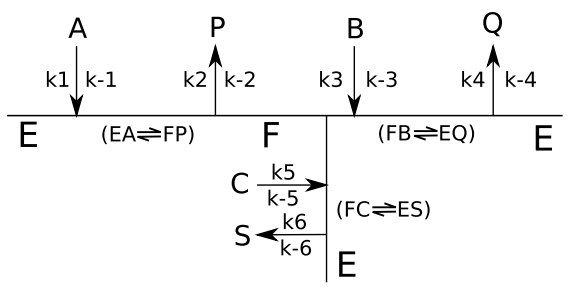

Figure 1: Cleland representation. The enzyme (E), the acyl enzyme $(\mathrm{F})$, the ester $(\mathrm{A})$, the $(R)$ and $(S)$ alcohols (respectively $\mathrm{B}$ and $\mathrm{C}$ ), the leaving alcohol product $(\mathrm{P})$, the $(R)$ and $(S)$ ester products (respectively $\mathrm{Q}$ and $\mathrm{S}$ ).

The kinetic profiles of multisubstrate systems can be resolved using the King-Altman method [20, 21], which, in the present work, has been devised as an interactive web form by BioKin Ltd. (available at: http://www.biokin.com/king-altman/). It was used to obtain the reaction velocity as a function of individual catalytic rate constants presented above in Cleland and King-Altman representations.

The model provides complex equations, whose detailed expression is given in the appendix. Equations were simplified by considering the system in the absence of products P, Q and S. Indeed, all reaction velocities were measured under conditions of initial rate of reaction, i.e. with negligible product concentrations. Thus, the forward velocities for the $R$ and $S$ ester products synthesis $\left(v_{\text {init. }}^{R}\right.$ and $v_{\text {init. }}^{R}$ ) were obtained: 


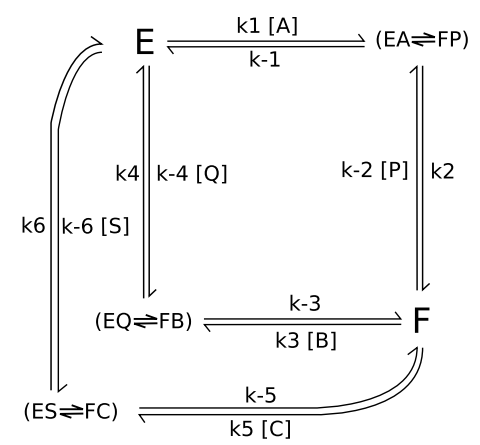

Figure 2: King-Altman representation. The enzyme (E), the acyl enzyme (F), the ester (A), the $(R)$ and $(S)$ alcohols (respectively $\mathrm{B}$ and $\mathrm{C})$, the leaving alcohol product $(\mathrm{P})$, the $(R)$ and $(S)$ ester products (respectively Q and $\mathrm{S})$.

$$
\begin{aligned}
& \frac{v_{\text {init. }}^{R}}{[E]_{t}}=\frac{n_{4}[A][B]}{d_{8}[A][C]+d_{9}[A][B]+d_{13}[C]+d_{14}[B]+d_{15}[A]} \\
& \frac{v_{\text {init. }}^{S}}{[E]_{t}}=\frac{n_{8}[A][C]}{d_{8}[A][C]+d_{9}[A][B]+d_{13}[C]+d_{14}[B]+d_{15}[A]}
\end{aligned}
$$

The complete formulas, with detailed values of $n_{i}$ and $d_{i}$ are reported in the appendix section.

\subsubsection{Kinetic parameter determination}

The Cleland nomenclature [22] allowed the calculation of the Michaelis-Menton constant $K_{M}$ for (R) and $(S)$ alcohols (respectively $K_{M}^{R}$ and $K_{M}^{S}$ ) and maximum reaction rates $V_{\max }^{R}$ and $V_{\max }^{S}$. The parameters determination was based on coefficients from the global equations 1 and 2.

$$
\begin{aligned}
\frac{V_{\max }^{R}}{[E]_{t}} & =\frac{k_{2} k_{4}}{k_{4}+k_{2}}=k_{c a t}^{R} & \frac{V_{\max }^{S}}{[E]_{t}} & =\frac{k_{2} k_{6}}{k_{6}+k_{2}}=k_{c a t}^{S} \\
K_{M}^{R} & =\frac{k_{2}\left(k_{-3}+k_{4}\right)}{k_{3}\left(k_{4}+k_{2}\right)} & K_{M}^{S} & =\frac{k_{2}\left(k_{-5}+k_{6}\right)}{k_{5}\left(k_{6}+k_{2}\right)}
\end{aligned}
$$

The four parameters $K_{M}^{R}, K_{M}^{R}, V_{\max }^{R}$ and $V_{\max }^{S}$ enabled the calculation of the enantiomeric ratio $E$, according to the following formula $E=\left(k_{c a t}^{R} / K_{M}^{R}\right) /\left(k_{c a t}^{S} / K_{M}^{S}\right)$ :

$$
E=\frac{k_{3} k_{4}\left(k_{-5}+k_{6}\right)}{k_{5} k_{6}\left(k_{-3}+k_{4}\right)}
$$

Thus, it appears that the $E$ value is not controlled by catalytic rate constants involved in the acylation step, the first part of the reaction $\left(k_{1}, k_{-1}, k_{2}, k_{-2}\right)$, (c.f. figures 1 and 2), indicating that the nature of the leaving alcohol did not influence the enantiomeric ratio $E$, through kinetic effects.

In addition, the ratio of initial reaction rates $v_{\text {init. }}^{R} / v_{\text {init. }}^{S}$ was equal to: 


$$
\frac{v_{\text {init. }}^{R}}{v_{\text {init. }}^{S}}=\frac{n_{4}[A][B]}{n_{6}[A][C]}=\frac{k_{3} k_{4}\left(k_{-5}+k_{6}\right)[B]}{k_{5} k_{6}\left(k_{-3}+k_{4}\right)[C]}
$$

Therefore, when reaction velocity is measured under conditions of initial rate, where B and C are enantiopure alcohols in racemic mixture, then the ratio of forward velocities $v_{\text {init. }}^{R} / v_{\text {init. }}^{S}$ is equal to:

$$
\frac{v_{\text {init. }}^{R}}{v_{\text {init. }}^{S}}=\frac{k_{3} k_{4}\left(k_{-5}+k_{6}\right)}{k_{5} k_{6}\left(k_{-3}+k_{4}\right)}=E
$$

Thus, the enantiomeric ratio $E$ is equal to the ratio $v^{R} / v^{S}$ in conditions of initial rate (insignificant concentration of products) and with a racemic mixture of alcohols at the initial step of the reaction. We can conclude that measuring the ratio of initial reaction rates $v^{R} / v^{S}$ is a valid method to determine the enantiomeric ratio $E$.

Similarly, the relationship between the ratio $v^{R} / v^{S}$ in conditions of initial reaction and $E$ was demontrated by Chen (1982) in the case of the simple Michaelis Menton model [11]. Furthermore, Chen's proposition remains correct in case of the Ping Pong Bi Bi system with $R$ or $S$ as competitive alcohol substrates.

We have confirmed here, then, that 1) $E$ values can be correctly determined by measuring the ratio of initial reaction rates for enantiopure ester synthesis, 2) $E$ values do not depend on catalytic rate constants involved in the acylation step.

\subsection{Molecular modeling results}

The results presented above suggest that there is an imprinting effect: the first substrate of the reaction and in particular the alkoxy part of the ester causes a conformation change of the enzyme, which is "memorized" by the enzyme and modifies its ability to discriminate between enantiomers of the second alcohol substrates. Interesting results, concerning the sensitivity of the enantioselectivity in relation to the enantiomeric form of the leaving alcohol, indicate that the imprinting effect involves modifications near the active site (table 1). Our attempts to confirm this hypothesis by molecular modeling are presented below.

Two representative structures (Clust1 and Clust2) were obtained from the cluster analysis of each system: free enzyme, enzyme $+R$ and enzyme $+S$ tetrahedral intermediates (TI- $R$ and TI- $S$ ). RMSD were calculated between them (table 2).

RMSD between two representative structures of the same system is usually lower than other values: $0.588 \AA$ for free enzyme, $0.700 \AA$ for enzyme-TI- $R, 0.658 \AA$ for enzyme-TI- $S$. Highest values were found when the representative structure of the enzyme-TI- $R$ was compared with other structures, RMSD reached $1.347 \AA$ when cluster 2 for the $R$ form and cluster 1 for the $\mathrm{S}$ form were compared.

Average structure superposition shows that the most important difference arises from the position of alpha helix 5, as shown in figure 3. The position of this helix differed between clusters for one examined enzyme structure, and also between $R$ and $S$ structures. 
Table 2: RMSD of the backbone between each representative structures of the three systems. Aligment was based on residues 35 to 80,100 to 240 and 260 to 290

\begin{tabular}{|c|c|c|c|c|c|c|c|}
\hline & \multicolumn{2}{|c|}{ Enz } & \multicolumn{2}{|c|}{ Enz+TI- $R$} & \multicolumn{2}{|c|}{ Enz+TI- $S$} \\
\hline & & Clust1 & Clust2 & Clust1 & Clust2 & Clust1 & Clust2 \\
\hline \multirow{2}{*}{ Enz } & Clust1 & 0 & 0.588 & 1.199 & 0.850 & 0.666 & 0.549 \\
\hline & Clust2 & & 0 & 0.970 & 1.120 & 0.724 & 0.649 \\
\hline \multirow{2}{*}{ Enz+TI- $R$} & Clust1 & & & 0 & 0.700 & 1.123 & 0.992 \\
\hline & Clust2 & & & & 0 & 1.347 & 1.233 \\
\hline \multirow{2}{*}{ Enz+TI- $S$} & Clust1 & & & & & 0 & 0.658 \\
\hline & Clust2 & & & & & & 0 \\
\hline
\end{tabular}

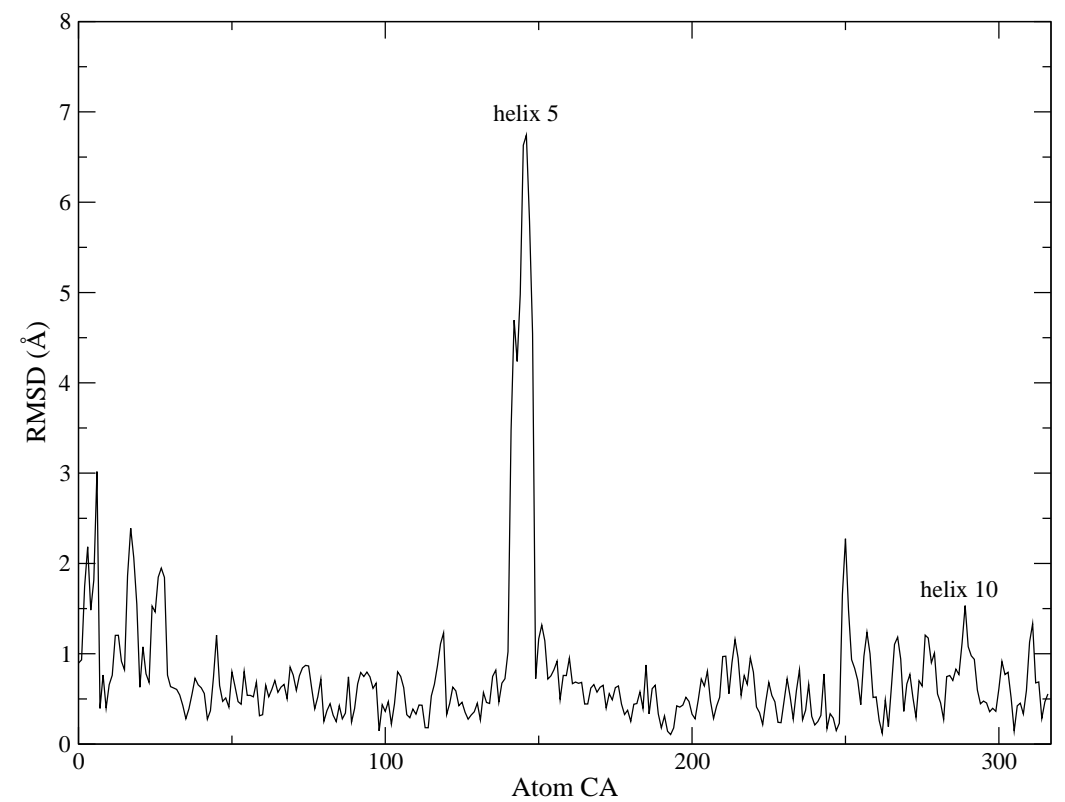

Figure 3: Measure of the deviation (in $\AA$ ) for the $\alpha$ carbons between the representative structure of the ten last nanoseconds between TI- $R$ and TI- $S$. 
Other authors have previously shown that the alpha helix 5 is highly mobile. Skjøt et al. [23] demonstrated that during a $10 \mathrm{~ns}$ dynamic trajectory in a water box, $\alpha$-helix 5 and 10 of CALB displayed significant mobilities. The RMSD matrix provides cluster results mainly based on the orientation of the $\alpha$ helix 5 . Here, $\alpha$ helix 5 orientation is not specific to the system studied, but similar mobility was observed for the three studied systems. Our conclusion is that specific differences in the global conformation of the enzyme between TI- $R$ and TI- $S$ are not observed.

In the second part of our study, we focused on the active site. Cluster analysis based on amino acids of the active site was done as described in the Computational Methods section. Amino acids alignment gave good superimposed structures, including side chains orientation (c.f. figure 4).

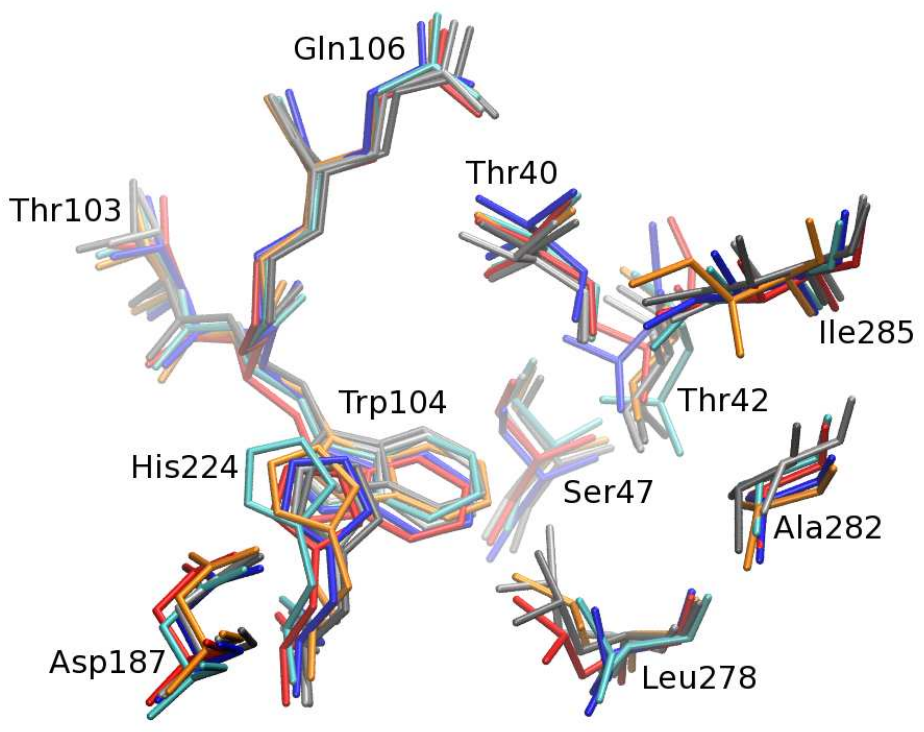

Figure 4: View of the active site for the six clusters obtained for the three studied systems after alignement based on the heavy atoms of residues 103,104,106, 224, 187, 40,42,47,278, 282, 285 and the backbone of residues 105 . The color code for the clusters is Clust1 for Enz-IT- $S$ in blue, Clust2 for Enz-IT- $S$ in light blue, Clust1 for Enz-IT- $R$ in red, Clust2 for Enz-IT- $R$ in orange, Clust1 for enzyme free in gray and Clust2 for enzyme free in dark gray.

Interestingly however, the orientation of the side chain of residue Ile285 was different for the cluster 2 of TI- $R$. This may be due to the specific constraint generated by the alcohol enantiomer on the side chain orientation which pointed toward Ile285. In the case of the $R$ enantiomer, the side chain of Ile285 rotated by 120 degrees in around 10 nanoseconds (figure 5). The cluster analysis was consistent with this fact, and split the trajectory into two dominant clusters, one before the rotation, and the second after it. Side chain orientation in the TI- $S$ system is the same as that observed with free enzyme. Residue Ile 285 belongs to $\alpha$ helix 10. Marton et al., demonstrated that mutations of residues Leu282 and Ile282 of the $\alpha$ helix 10 affected enantioselectivity [24]. Thus, side chain rotation of Ile285 may also influence the enantioselectivity of the 
reaction.

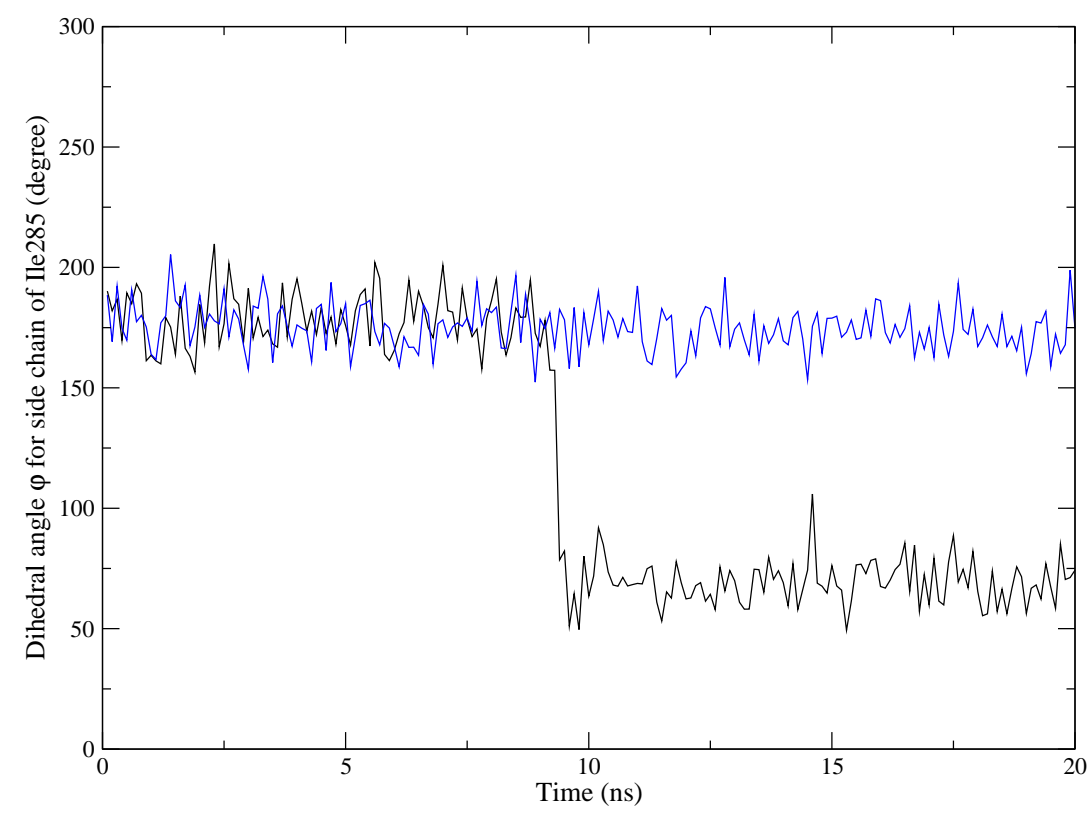

Figure 5: Diheral angle $\varphi$ defining the side chain rotation of residue Ile285. In back pour the $R$ enantiomer and in blue for the $S$ enantiomer.

A major question concerns the timescale of side chain rotation, such as of the branched-chain of Ile285. The time scale for the rotation of a buried side chain can be very large $\left(10^{-4}\right.$ to $\left.1 \mathrm{~s}\right)$ [25]. Experimental results show that buried side chains rotate very slowly compared with the time scale of molecular dynamics. NMR was used by Skrynnikov et al. [26] to quantitate slow hydrogen-deuterium exchange processes at methylcontaining side chains in proteins. This method was also applied to the study of ms time scale side-chain dynamics of methionine residues in a buried cavity. These authors observed that the methionine residues were sensitive to an exchange event with a rate of the order of $1200 \mathrm{~s}^{-1}$ at $20^{\circ} \mathrm{C}$ and that the corresponding motions may be linked to a process which allows entry and exit of ligands to and from the cavity. Similiar NMR studies on a protease, by Ishima et al. [27], demonstrated that the hydrogen-deuterium exchange time of buried methyl side chains was above $1 \mathrm{~ms}$.

The side chain of residue Ile285 is partially buried, as it is oriented toward the top of the stereospecifity pocket in the active site, and near the side chain of the alcohol. The time scale of Ile285 side chain rotation can thus be considered to be around $1 \mathrm{~ms}$. Previously the same order of magnitude was obtained for pentan-2-ol transesterification in a solid-gas reactor [18]: $k_{c a t}$ equal to $800 \mathrm{~s}^{-1}$ and $17 \mathrm{~s}^{-1}$ for (R)- and $(S)$ pentan-2-ol respectively. The similarity of these two time scales namely, side chain rotation and substrate catalysis, is consistent with the hypothesis of "imprinting effect". Ile285 side chain rotation provides a much more suitable active site shape for interacting with the substrate $(R)$ - enantiomer alcohol. This is 
exactly what is experimentally observed: enantiopreference for the $R$ form of pentan-2-ol increases when the (R)-1-methylpentyl propanoate is used as acyl donor.

Other authors also performed molecular modeling studies to explore enzyme structural changes upon imprinting. Rich and Dordick [28] obtained an increase of subtilisin catalytic rate and also a better control of enzyme substrate specificity, by means of lyophilizing subtilisin in presence of different nucleophile substrates, as "imprinters". By molecular dynamic simulations it was shown that structural changes in the catalytic triad occurred during imprinting, that may contribuate to imprinting-induced substrate selectivity.

\section{Conclusion}

Experimental results shown here demonstrate that using an ester with an adequate alkoxy group is an efficient method to enhance enantioselectivity. Generally, an alkoxy group larger than ethyl increased enantioselectivity. Furthermore, the resolution of pentan-2-ol was sensitive to the chirality of the alkoxy group of the ester. Thus, $(R)$-1-methylpentyl propanoate increased enantioselectivity compared to the racemic mixture, whereas $S$ enantiomer decreased enantioselectivity compared with the racemic mixture.

The comprehensive study of the full kinetics for the Ping Pong Bi Bi mechanism, with three substrates, one ester and two competitive $R$ and $S$ alcohols, allowed us to confirm that the experimental method, based on initial rate measurements employed here for the determination of enantiomeric ratio, is relevant. In particular, it excluded the hypothesis that enantiomeric ratio modifications observed in the experimental results could arise from a kinetic model pitfall.

Finally, molecular dynamics simulations were performed to discriminate between conformational changes caused by both $(R)$ - and $(S)$ - enantiopure 1-methylpropyl propanoate. It appears that the $R$ enantiomer causes the rotation of the side chain of residue Ile285, which appears to have an effect on subsequent discrimination between secondary alcohol enantiomers. If this is the general case, molecular imprinting by the first substrate would offer the possibility of controlling enantioselectivity for a second substrate and thus provide a new tool for biocatalyst engineering.

\section{Acknowledgements}

This study was supported by the French ANR (National Research Agency) through the EXPENANTIO project (program CP2D). CINES and IDRIS from GENCI (Grand Equipement National de Calcul Intensif) are acknowledged for giving access to super-computing facilities. The manuscript was corrected by a native English speaking scientific translator (http://traduction.lefevere-laoide.net) 


\section{References}

[1] M. T. Reetz, Curr. Opin. Chem. Biol. 6 (2002) 145-150.

[2] J.-Y. J.-Y. Yan, Y.-J. Yan, J. Yang, L. Xu, Y. Liu, Proc. Biochem. 44 (2009) 1128-1132.

[3] J. Yang, L. Liu, X. Cao, Enzyme Microb. Technol. 46 (2010) 257-261.

[4] G. Hellner, Z. Boros, A. Tomin, L. Poppe, Adv. Synth. Catal. 353 (2011) 2481-2491.

[5] D. Lee, Y. K. Choi, M.-J. Kim, Org. Lett. 2 (2000) 2553-2555.

[6] D. Lousa, A. M. Baptista, C. M. Soares, Protein Sci. 20 (2011) 379-386.

[7] J. C. Rotticci-Mulder, M. Gustavsson, M. Holmquist, K. Hult, M. Martinelle, Protein Expres. Purif. 21 (2000) 386-392.

[8] A. Ducret, M. Trani, R. Lortie, Enzyme Microb. Technol. 22 (1998) 212-216.

[9] V. Leonard, S. Lamare, M.-D. Legoy, M. Graber, J. Mol. Catal. B: Enzym. 32 (2004) 53-59.

[10] F. Létisse, S. Lamare, M.-D. Legoy, M. Graber, Biochim. Biophys. Acta 1652 (2003) 27-34.

[11] C.-S. Chen, Y. Fujimoto, G. Girdaukas, C. J. Sih, J. Am. Chem. Soc. 104 (1982) 7294-7299.

[12] V. Leonard-Nevers, Z. Marton, S. Lamare, K. Hult, M. Graber, J. Mol. Catal. B: Enzym. 59 (2009) 90-95.

[13] J. Uppenberg, M. T. Hansen, S. Patkar, T. A. Jones, Structure 2 (1994) 293-308.

[14] N. Otte, M. Bocola, W. Thiel, J. Comput. Chem. 30 (2009) 154-62.

[15] W. Humphrey, A. Dalke, K. Schulten, J. Molec. Graphics 14 (1996) 33-38.

[16] J. H. Ward, J. Am. Stat. Assoc. 58 (1963) 236-244.

[17] R Development Core Team, R: A Language and Environment for Statistical Computing, R Foundation for Statistical Computing, Vienna, Austria, ISBN 3-900051-07-0 (2011).

URL http://www.R-project.org

[18] L. Chaput, Y.-H. Sanejouand, A. Balloumi, V. Tran, M. Graber, J. Mol. Catal. B: Enzym. (accepted).

[19] J. González-Sabína, V. Gotor, F. Rebolledo, Tetrahedron: Asymmetry 15 (2004) 481-488.

[20] E. L. King, C. Altman, J. Phys. Chem. 60 (1956) 1375-1378.

[21] I. H. Segel, Enzyme kinetics, Wiley, New York, 1993, pp. 606-625. 
334 [22] W. Cleland, Biochim. Biophys. Acta 67 (1963) 104-137.

[23] M. Skjøt, L. De Maria, R. Chatterjee, A. Svendsen, S. A. Patkar, P. R. Østergaard, J. Brask, ChemBioChem 10 (2009) 520-527.

[24] Z. Marton, V. Léonard-Nevers, P.-O. Syrén, C. Bauer, S. Lamare, K. Hult, V. Tran, M. Graber, J. Mol. Catal. B: Enzym. 65 (2010) 11-17.

[25] S. J. Benkovic, S. Hammes-Schiffer, Science 301 (2003) 1196-1202.

[26] N. R. Skrynnikov, F. A. A. Mulder, B. Hon, F. W. Dahlquist, L. E. Kay, J. Am. Chem. Soc. 123 (2001) $4556-4566$.

[27] R. Ishima, J. M. Louis, D. A. Torchia, J. Am. Chem. Soc. 121 (1999) 11589-11590.

[28] J. O. Rich, J. S. Dordick, J. Am. Chem. Soc. 119 (1997) 3245-3252. 


\section{Appendix}

Speed rate equation for synthesis of $R$ and $S$ enantiomer products $v^{R}$ and $v^{S}$ :

$$
\begin{gathered}
v^{R}=d[Q] / d t=k_{+4}[F B]-k_{-4}[Q][E] \\
v^{S}=d[S] / d t=k_{+6}[F C]-k_{-6}[S][E] \\
\frac{v^{R}}{[E]_{t}}=\frac{N_{R}}{D} \\
\frac{v^{S}}{[E]_{t}}=\frac{N_{S}}{D}
\end{gathered}
$$

346 Expression of numerators and denominator, $N_{R}, N_{S}$, and $D$ :

$$
\begin{gathered}
N_{R}=+n_{1}[Q][P]+n_{2}[C][Q]+n_{3}[B][S]+n_{4}[A][B] \\
N_{S}=+n_{5}[S][P]+n_{6}[C][Q]+n_{7}[B][S]+n_{8}[A][C] \\
D=+d_{1}[P][S]+d_{2}[Q][P]+d_{3}[C][S]+d_{4}[C][Q]+d_{5}[B][S]+d_{6}[B][Q]+d_{7}[A][P] \\
+d_{8}[A][C]+d_{9}[A][B]+d_{10}[S]+d_{11}[P]+d_{12}[Q]+d_{13}[C]+d_{14}[B]+d_{15}[A] \\
n_{1}=-k_{-1} k_{-2} k_{-3} k_{-4} k_{-5}-k_{-1} k_{-2} k_{-3} k_{-4} k_{+6} \\
n_{2}=-k_{-1} k_{-3} k_{-4} k_{+5} k_{+6}-k_{+2} k_{-3} k_{-4} k_{+5} k_{+6} \\
n_{3}=+k_{-1} k_{+3} k_{+4} k_{-5} k_{-6}+k_{+2} k_{+3} k_{+4} k_{-5} k_{-6} \\
n_{5}=-k_{-1} k_{-2} k_{-3} k_{-5} k_{-6}-k_{-1} k_{-2} k_{+4} k_{-5} k_{-6}
\end{gathered}
$$




$$
\begin{gathered}
n_{6}=+k_{-1} k_{-3} k_{-4} k_{+5} k_{+6}+k_{+2} k_{-3} k_{-4} k_{+5} k_{+6} \\
n_{7}=-k_{-1} k_{+3} k_{+4} k_{-5} k_{-6}-k_{+2} k_{+3} k_{+4} k_{-5} k_{-6} \\
n_{8}=+k_{+1} k_{+2} k_{-3} k_{+5} k_{+6}+k_{+1} k_{+2} k_{+4} k_{+5} k_{+6} \\
d_{1}=k_{-2} k_{+4} k_{-5} k_{-6}+k_{-2} k_{-3} k_{-5} k_{-6}+k_{-1} k_{-2} k_{+4} k_{-6}+k_{-1} k_{-2} k_{-3} k_{-6} \\
d_{2}=k_{-2} k_{-3} k_{-4} k_{+6}+k_{-2} k_{-3} k_{-4} k_{-5}+k_{-1} k_{-2} k_{-4} k_{+6}+k_{-1} k_{-2} k_{-4} k_{-5} \\
d_{10}=k_{-1} k_{-2} k_{+4} k_{+6}+k_{-1} k_{-2} k_{+4} k_{-5}+k_{-1} k_{-2} k_{-3} k_{+6}+k_{-1} k_{-2} k_{-3} k_{-5} \\
d_{3}=k_{-1} k_{+4} k_{+5} k_{-6}+k_{-1} k_{-3} k_{+5} k_{-6}+k_{+2} k_{+4} k_{+5} k_{-6}+k_{+2} k_{-3} k_{+5} k_{-6} \\
d_{7}=k_{+1} k_{+3} k_{+4} k_{+6}+k_{+1} k_{+3} k_{+4} k_{-5}+k_{+1} k_{+2} k_{+3} k_{+5} k_{+6}+k_{+1} k_{+2} k_{+3} k_{-5} k_{+5} k_{+6}+k_{+1} k_{-3} k_{+5} k_{+6}+k_{+5} k_{-6} \\
d_{4}=k_{-1} k_{-4} k_{+5} k_{+6}+k_{+2} k_{-4} k_{+5} k_{+6}+k_{-1} k_{-3} k_{-4} k_{+5}+k_{+2} k_{-3} k_{-4} k_{+5} k_{+5}+k_{+1} k_{-2} k_{-3} k_{+6}+k_{+1} k_{-2} k_{-3} k_{-5} k_{+6}+k_{-1} k_{+3} k_{-4} k_{-5}+k_{+2} k_{+3} k_{-4} k_{+6}+k_{+2} k_{+3} k_{-4} k_{-5} \\
d_{5}=k_{-1} k_{+3} k_{-5} k_{-6}+k_{+2} k_{+3} k_{-5} k_{-6}+k_{-1} k_{+3} k_{+4} k_{-6}+k_{+2} k_{+3} k_{+4} k_{-6}
\end{gathered}
$$




$$
\begin{aligned}
& d_{12}=k_{-1} k_{-3} k_{-4} k_{+6}+k_{-1} k_{-3} k_{-4} k_{-5}+k_{+2} k_{-3} k_{-4} k_{+6}+k_{+2} k_{-3} k_{-4} k_{-5} \\
& d_{13}=k_{-1} k_{+4} k_{+5} k_{+6}+k_{-1} k_{-3} k_{+5} k_{+6}+k_{+2} k_{+4} k_{+5} k_{+6}+k_{+2} k_{-3} k_{+5} k_{+6} \\
& d_{14}=k_{-1} k_{+3} k_{+4} k_{+6}+k_{-1} k_{+3} k_{+4} k_{-5}+k_{+2} k_{+3} k_{+4} k_{+6}+k_{+2} k_{+3} k_{+4} k_{-5} \\
& d_{15}=k_{+1} k_{+2} k_{+4} k_{+6}+k_{+1} k_{+2} k_{+4} k_{-5}+k_{+1} k_{+2} k_{-3} k_{+6}+k_{+1} k_{+2} k_{-3} k_{-5}
\end{aligned}
$$

